\title{
Dark excitons and the elusive valley polarization in transition metal dichalcogenides
}

\author{
M Baranowski ${ }^{1,2} \dagger$, A Surrente ${ }^{1} \dagger$, D K Maude ${ }^{1}$, M Ballottin ${ }^{3}$, A \\ A Mitioglu ${ }^{3}$, P C M Christianen ${ }^{3}$, Y C Kung ${ }^{4}$, D Dumcenco ${ }^{4}$, \\ A Kis ${ }^{4}$ and $\mathbf{P}$ Plochocka ${ }^{1}$ \\ ${ }^{1}$ Laboratoire National des Champs Magnétiques Intenses, UPR 3228, \\ CNRS-UGA-UPS-INSA, Grenoble and Toulouse, France \\ ${ }^{2}$ Department of Experimental Physics, Faculty of Fundamental Problems of \\ Technology, Wroclaw University of Science and Technology, Wroclaw, Poland \\ ${ }^{3}$ High Field Magnet Laboratory (HFML - EMFL), Radboud University, 6525 ED \\ Nijmegen, The Netherlands \\ ${ }^{4}$ Electrical Engineering Institute and Interdisciplinary Center for Electron \\ Microscopy (CIME), École Polytechnique Fédérale de Lausanne (EPFL), CH-1015 \\ Lausanne, Switzerland \\ E-mail: paulina.plochocka@lncmi.cnrs.fr
}

November 2016

\begin{abstract}
A rate equation model for the dark and bright excitons kinetics is proposed which explains the wide variation in the observed degree of circular polarization of the PL emission in different TMDs monolayers. Our work suggests that the dark exciton states play an important, and previously unsuspected role in determining the degree of polarization of the PL emission. A dark exciton ground state provides a robust reservoir for valley polarization, which tries to maintain a Boltzmann distribution of the bright exciton states in the same valley via the intra valley bright dark exciton scattering mechanism. The dependence of the degree of circular polarization on the detuning energy of the excitation in $\mathrm{MoSe}_{2}$ suggests that the electron-hole exchange interaction dominates over two LA phonon emission mechanism for inter valley scattering in TMDs.
\end{abstract}

Submitted to: 2D Mater.

$\dagger$ these authors contributed equally to this work 


\section{Introduction}

Monolayer transition metal dichalcogenides (TMDs) have attracted tremendous interest due to their unique electrical and optical properties that are absent in their bulk and fewlayer forms [1, 2, 3, 4, 5]. Bulk TMDs such as $\mathrm{MoS}_{2}, \mathrm{MoSe}_{2}, \mathrm{WS}_{2}$ and $\mathrm{WSe}_{2}$ are indirect band gap semiconductors with the conduction band minimum located half way between the $\mathbf{K}$ and $\boldsymbol{\Gamma}$ points of the Brillouin zone, while the valence band maximum is located at the $\boldsymbol{\Gamma}$ point. Monolayer TMDs have a direct band gap with the conduction and valence band minima located at the the $\mathbf{K}$ point of the Brillouin zone [6, 7, 8, 5]. The direct gap in the visible light energy range makes single-layer TMDs very attractive materials for optoelectronic devices such as light emitting diodes and detectors [9, 10, 11, 1, 12, 13. Moreover, monolayer TMDs possess a unique band structure that makes them ideal material systems for valleytronics [14, 15, 2, 16].

The band-edges in monolayer TMDs are located at the degenerate but non equivalent $\mathbf{K}$ and $\mathbf{K}^{\prime}$ corners of the hexagonal Brillouin zone. The valence band electrons in $\mathbf{K}$ and $\mathbf{K}^{\prime}$ valleys have non zero magnetic moment related to hybridization of the $d_{x^{2}-y^{2}}$ and $d_{x y}$ orbitals [17]. Broken inversion symmetry together with time reversal symmetry lead to magnetic moments in the $\mathbf{K}$ and $\mathbf{K}^{\prime}$ valleys with opposite directions which results in valley dependent optical selection rules. The $\mathbf{K}$ and $\mathbf{K}^{\prime}$ valleys can be selectively excited by $\sigma^{ \pm}$circularly polarized photons, respectively [18]. In addition, the strong spin-orbit interaction in TMDs lifts the degeneracy of spin states particularly in the valence band giving rise to well separated A and B-excitonic transitions in absorption or reflectance spectra [6, 19, 20, 21. The valence band splitting in TMDs is in the range of a few-hundred meV [3, [5]. In the conduction band this splitting is much smaller and is in the range of few to tens meV [5, 22, Spin-orbit interaction in combination with the broken time reversal symmetry leads to the coupling of spin states and valleys indexes (spin states are reversed in $\mathbf{K}$ and $\mathbf{K}^{\prime}$ ) [15].

The spin-orbit interaction in the conduction band lifts the degeneracy of bright and dark exciton states of the $\mathrm{A}$ and $\mathrm{B}$-excitons. In figure 1 the possible spin configurations of the A-exciton in $\mathrm{MoX}_{2}$ and $\mathrm{WX}_{2}(\mathrm{X}=\mathrm{S}, \mathrm{Se})$ are indicated schematically. Here, and in the following, we neglect the B-excitons which do not contribute to PL emission due to the large spin-orbit splitting of the valence band. The schematic is based on both experiments [23, 24] and theoretical calculations [17, 22, 5] which show that Molybdenum based TMDs have a bright A-exciton ground state while Tungsten based TMDs have a dark A-exciton ground state due to the reversal of the spin levels in the conduction band (the situation is reversed for the B-excitons).

Because of valley spin-locking, the large distance (in $\mathbf{k}$ space) between the $\mathbf{K}$ and $\mathbf{K}^{\prime}$ valleys and the significant valence band splitting, it is expected that the exciton scattering between valleys will be strongly suppressed and robust valley polarization can be achieved. Experimentally, a significant degree of circular polarization of photoluminescence (PL) after circularly polarized excitation has been observed in $\mathrm{MoS}_{2}$, $\mathrm{WS}_{2}$ and $\mathrm{WSe}_{2}$ [25, 26, 27, 28, 29, 30, 31]. In addition, coherent emission from $\mathbf{K}$ and $\mathbf{K}^{\prime}$ 

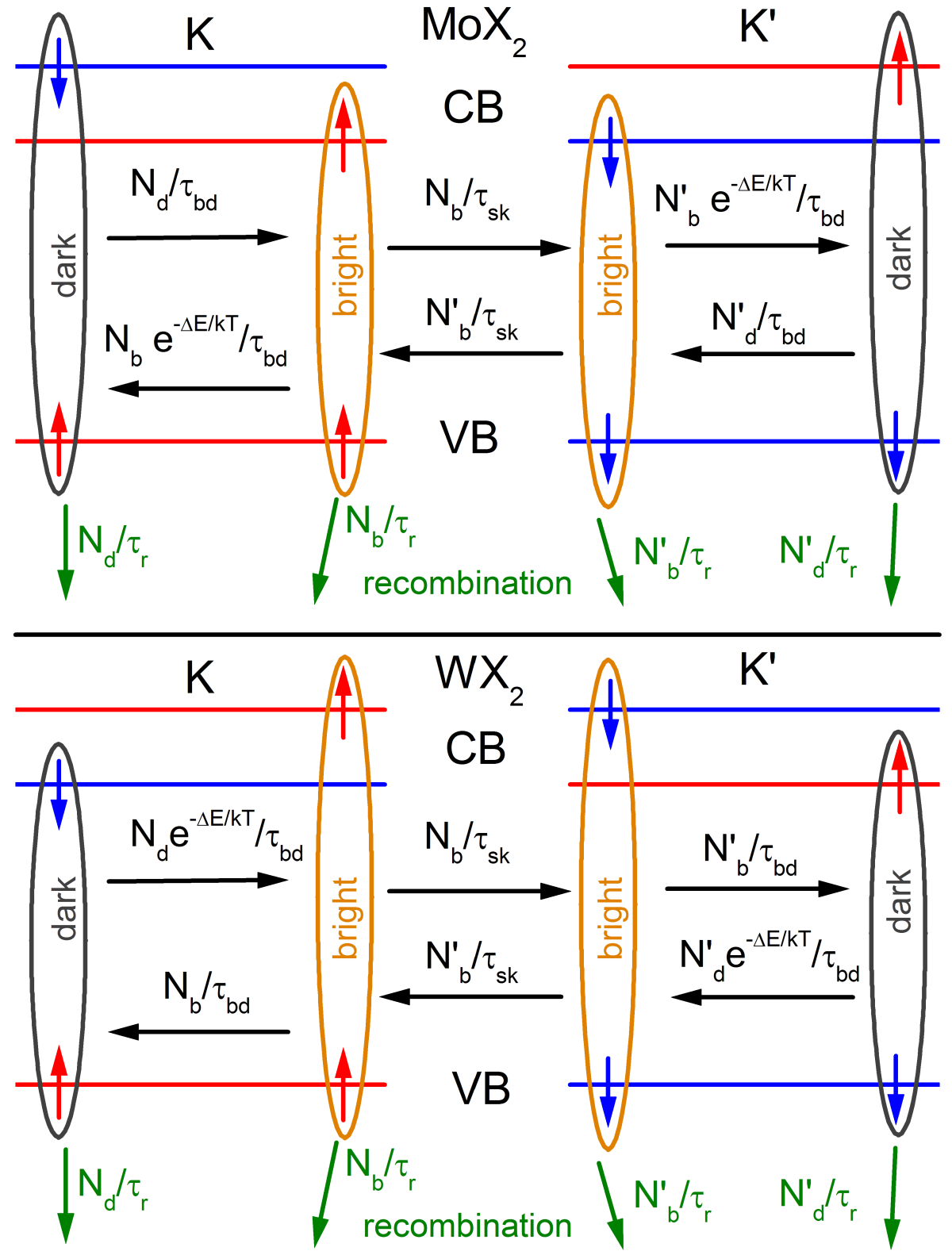

Figure 1. Schematic showing the band structure and spin configuration of the bright and dark $\mathrm{A}$-excitons in $\mathrm{MoX}_{2}$ and $\mathrm{WX}_{2}$ materials. The possible inter and intra valley scattering and recombination paths included in the rate equations (see text) are indicated schematically. The influence of B-excitons can safely be neglected due to the large spin-orbit splitting in the valence band.

valleys was observed in $\mathrm{WSe}_{2}$ [32]. These results demonstrate that valley polarization persists for a sufficiently long time to be observed in the polarization resolved PL. However, there are some inconsistencies in this picture.

Despite the very similar band structure of $\mathrm{WX}_{2}$ and $\mathrm{MoX}_{2}$ monolayers, it is much easier to achieve polarized emission in Tungsten based TMDs (see for example [30]). In particular, obtaining polarized $\mathrm{PL}$ emission in $\mathrm{MoSe}_{2}$ proved to be extremely difficult, requiring almost resonant excitation (detuning $\sim 70 \mathrm{meV}$ ) and gave only $20 \%$ 
circular polarization [33]. Another puzzling aspect is that in $\mathrm{WX}_{2}$ monolayers the $\mathrm{PL}$ polarization has only a weak dependence [32, 30, 34] on the excitation photon energy while in $\mathrm{MoX}_{2}$ the dependence is more pronounced [30, 31, 33, 26]. These observations are summarized in figure 2(a), where the reported values of the degree of circular polarization for various detunings of the excitation are shown. It is clearly visible that for large value of detuning the $\mathrm{PL}$ polarization is achieved only for $\mathrm{WX}_{2}$. For detuning in the range of $100-150 \mathrm{meV}$ only $\mathrm{MoS}_{2}$ exhibits significant degree of circular polarization in case of Molybdenum based materials.

Here we show that the achievable degree of circular polarization is directly linked with the alignment of bright and dark exciton states in Mo and $\mathrm{W}$ based monolayer TMDs. We propose a simple rate equation model that demonstrates that the different alignment of excitonic states can enhance the degree of PL circular polarization which is limited by the inter-valley scattering. Strong support for this model is provided by a detailed excitation energy dependent investigation of the degree of circular polarization of the PL emission in $\mathrm{MoSe}_{2}$ for detunings as small as $20 \mathrm{meV}$.

\section{Model}

Despite the large valence band splitting and spin-valley locking, ultrafast optical spectroscopy experiments show that the inter-valley scattering is very effective in TMDs and the valley depolarization occurs in the range of few to several picoseconds [36, 38, 39, 40, 41]. There are different propositions in literature explaining exciton scattering between valleys.

One of the proposed mechanisms involves the thermalization of photo-created electron-hole pairs via the emission of two longitudinal acoustic (LA) phonons [31, 33, 16]. Provided that exciton kinetic energy is above the energy of two LA phonons at the $\mathbf{K}$ point, the exciton can be scattered between the $\mathbf{K}$ and $\mathbf{K}^{\prime}$ valleys. Two phonon emission is required for the simultaneous scattering of the electron and hole. The LA phonon model is particularly appealing as it provides a relatively straightforward way to describe the degree of PL circular polarization as a function of the excitation energy. The larger LA phonon energy in $\mathrm{MoS}_{2}(30 \mathrm{meV})$ [42] compared to $\mathrm{MoSe}_{2}(19 \mathrm{meV})$ [3] is consistent with the significant degree of circular polarization of the PL emission observed in $\mathrm{MoS}_{2}$ for larger detuning of the excitation than in $\mathrm{MoSe}_{2}$. However, the LA phonon model is unable to explain the different degrees of polarization observed in $\mathrm{MoX}_{2}$ and $\mathrm{WX}_{2}$. The LA phonon energy of $\mathrm{WS}_{2}$ and $\mathrm{WSe}_{2}$ at the $\mathbf{K}$ point are respectively $24 \mathrm{meV}$ [42] and $18 \mathrm{meV}$ [44]. Therefore, we would expect that the observation of polarized PL emission in $\mathrm{WX}_{2}$ should be extremely difficult (as in $\mathrm{MoSe}_{2}$ ), while experimentally polarized emission is easily achieved.

An alternative explanation for the valley depolarization is related to electron-hole

exchange interaction [45, 46]. This mechanism can be considered as the simultaneous virtual recombination of a bright exciton in the $\mathbf{K}$ valley and the creation of a bright exciton in the $\mathbf{K}^{\prime}$ valley [45]. Theoretically, the effectiveness of this mechanism depends 


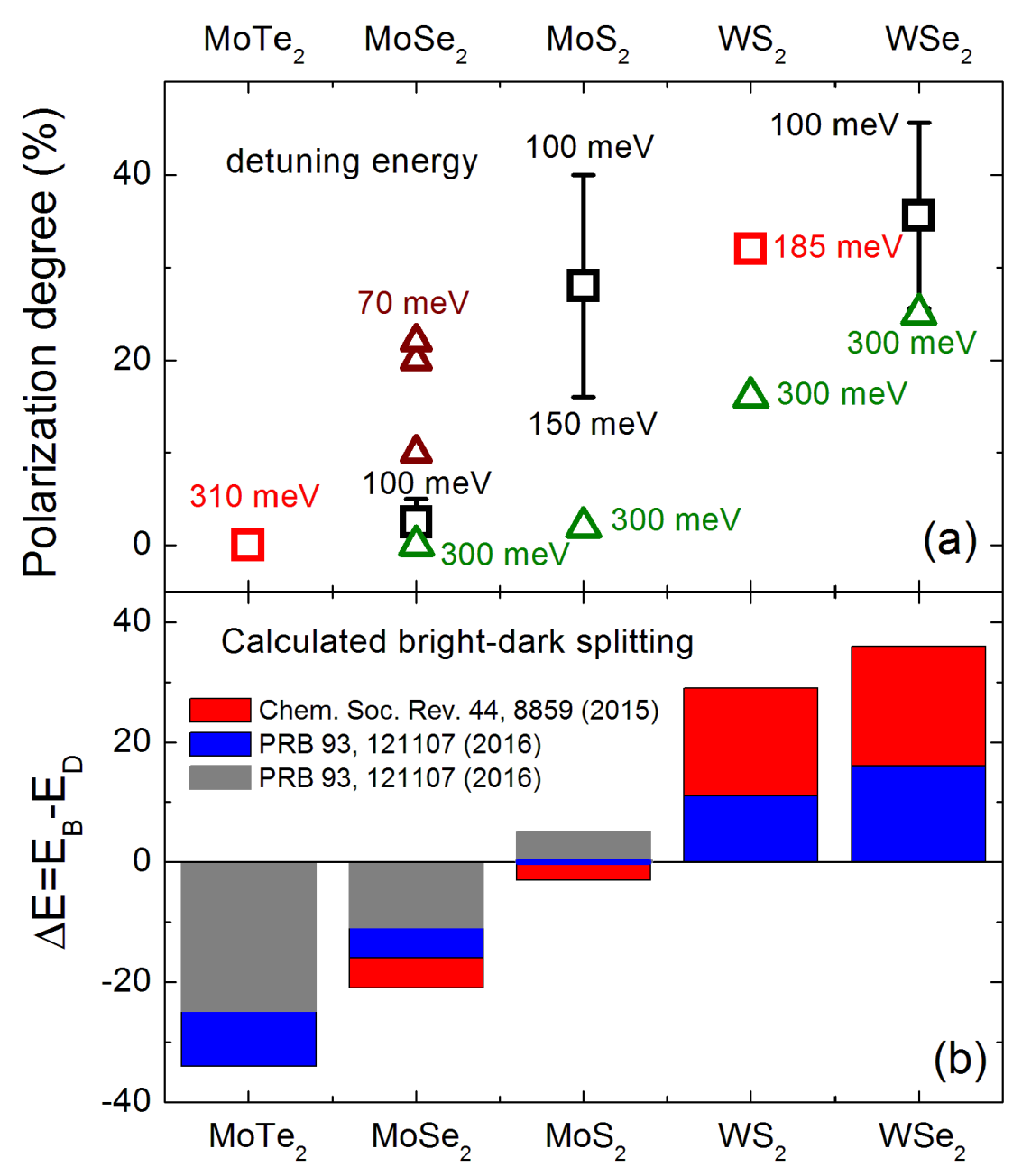

Figure 2. (a) Reported literature values of PL circular polarization degree for TMDs [35, 26, 32, 28, 29, 30, 34, 27, 31, 25, 36, 33, 37]. The open squares are average values for detuning range of $100-150 \mathrm{meV}$ and error bars represent maximum and minimum of reported values. Points with different value of detuning are labeled accordingly. (b) Literature data for the calculated difference between bright and dark exciton state energy [5, 22].

on the exciton center of mass momentum [45]. The scattering is faster when the exciton has large kinetic energy, which in principle explains the dependence of the degree of circular polarization of the PL emission on the energy of the excitation. The differences in the degree of circular polarization in different materials can be attributed to different material constants. However, in figure 2 there is a clear correlation between the size of the bright and dark exciton splitting and the obtained degree of polarization. This suggests that a dark exciton ground state favors polarized emission, even for non resonant excitation.

In the simplest approach, the kinetics of valley polarization can be described by 
two coupled rate equations [31]:

$$
\left\{\begin{array}{l}
\frac{\mathrm{d} N}{\mathrm{~d} t}=-\frac{N}{\tau_{s k}}-\frac{N}{\tau_{r}}+\frac{N^{\prime}}{\tau_{s k}}+g \\
\frac{\mathrm{d} N^{\prime}}{\mathrm{d} t}=-\frac{N^{\prime}}{\tau_{s k}}-\frac{N^{\prime}}{\tau_{r}}+\frac{N}{\tau_{s k}}+g^{\prime},
\end{array}\right.
$$

where $N$ and $N^{\prime}$ are the exciton populations in the $\mathbf{K}$ and $\mathbf{K}^{\prime}$ valleys, $\tau_{s k}$ is the intervalley (exchange) scattering time, $\tau_{r}$ is the recombination time of exciton (including both radiative and nonradiative recombination channels) and $g$ and $g^{\prime}$ are generation rates of excitons in the $\mathbf{K}$ and $\mathbf{K}^{\prime}$ valley. In this model the time dependent degree of the PL circular polarization $P(t)$ is given by

$$
P(t)=\frac{N(t)-N(t)^{\prime}}{N(t)+N(t)^{\prime}}
$$

For cw excitation ( $g=$ const or $g^{\prime}=$ const) after some time the population of excitons in the $\mathbf{K}$ and $\mathbf{K}^{\prime}$ valleys, and thus the degree of circular polarization, are constant. In the following we consider only steady state conditions and we will not discuss the time dependence of $P$.

Including the effect of exciton scattering between bright and dark states requires four coupled rate equations. It is important to note that the exchange interactions can scatter excitons only between bright exciton states in the $\mathbf{K}$ and $\mathbf{K}^{\prime}$ valleys [45]. We assume, as before, that the inter-valley bright exciton scattering is described by the time constant $\tau_{s k}$. A term is added to describe the intra-valley scattering between bright and dark states, which has been observed experimentally [24, 23], notably in the temperature dependence of PL intensity which depends on the alignment of bright and dark exciton states. The PL in $\mathrm{MoS}_{2}$ and $\mathrm{MoSe}_{2}$ is quenched with increasing temperature while in $\mathrm{WSe}_{2}$ the $\mathrm{PL}$ is initially enhanced with increasing temperature. This observation shows that the alignment of the bright and dark exciton states has significant impact on the exciton recombination kinetics. Moreover, the transient absorption spectroscopy experiments demonstrate that carriers experience very effective intra-valley scattering with simultaneous spin flip process [47, 48] which can also lead to the population of dark excitons states. In the $\mathrm{WX}_{2}$ TMDs the scattering rate from dark to bright states is described by $\frac{1}{\tau_{b d}} \exp \left(-\frac{\Delta E}{k T}\right)$ while in MoX $_{2}$ TMDs the scattering rate is equal to $\frac{1}{\tau_{b d}}$. Scattering from bright to dark states are described by $\frac{1}{\tau_{b d}}$ and $\frac{1}{\tau_{b d}} \exp \left(-\frac{\Delta E}{k T}\right)$ for $\mathrm{WX}_{2}$ and $\mathrm{MoX}_{2}$, respectively. The Boltzmann factor reflects the experimentally observed energy barrier $\Delta E$ (the absolute value of bright-dark exciton state splitting) for scattering between bright and dark states [24]. This factor is responsible for the very different exciton recombination kinetics depending upon the nature of the ground state. Since in $\mathrm{WX}_{2}$ the transition from bright to dark state is downwards it is effective while in $\mathrm{MoX}_{2}$ this transition is damped due to the energy barrier. The system of rate 
equations for $\mathrm{WX}_{2}$ materials can be written as follows,

$$
\left\{\begin{array}{l}
\frac{\mathrm{d} N_{b}}{\mathrm{~d} t}=-\frac{N_{b}}{\tau_{s k}}-\frac{N_{b}}{\tau_{r}}-\frac{N_{b}}{\tau_{b d}}+\frac{N_{b}^{\prime}}{\tau_{s k}}+\frac{N_{d}}{\tau_{b d}} \exp \left(-\frac{\Delta E}{k T}\right)+g \\
\frac{\mathrm{d} N_{b}^{\prime}}{\mathrm{d} t}=-\frac{N_{b}^{\prime}}{\tau_{s k}}-\frac{N_{b}^{\prime}}{\tau_{r}}-\frac{N_{b}^{\prime}}{\tau_{b d}}+\frac{N_{b}}{\tau_{s k}}+\frac{N_{d}^{\prime}}{\tau_{b d}} \exp \left(-\frac{\Delta E}{k T}\right)+g^{\prime} \\
\frac{\mathrm{d} N_{d}}{\mathrm{~d} t}=-\frac{N_{d}}{\tau_{r}}-\frac{N_{d}}{\tau_{b d}} \exp \left(-\frac{\Delta E}{k T}\right)+\frac{N_{b}}{\tau_{b d}} \\
\frac{\mathrm{d} N_{d}^{\prime}}{\mathrm{d} t}=-\frac{N_{d}^{\prime}}{\tau_{r}}-\frac{N_{d}^{\prime}}{\tau_{b d}} \exp \left(-\frac{\Delta E}{k T}\right)+\frac{N_{b}^{\prime}}{\tau_{b d}}
\end{array}\right.
$$

and for $\mathrm{MoX}_{2}$ it has the following form:

$$
\left\{\begin{array}{l}
\frac{\mathrm{d} N_{b}}{\mathrm{~d} t}=-\frac{N_{b}}{\tau_{s k}}-\frac{N_{b}}{\tau_{r}}-\frac{N_{b}}{\tau_{b d}} \exp \left(-\frac{\Delta E}{k T}\right)+\frac{N_{b}^{\prime}}{\tau_{s k}}+\frac{N_{d}}{\tau_{b d}}+g \\
\frac{\mathrm{d} N_{b}^{\prime}}{\mathrm{d} t}=-\frac{N_{b}^{\prime}}{\tau_{s k}}-\frac{N_{b}^{\prime}}{\tau_{r}}-\frac{N_{b}^{\prime}}{\tau_{b d}} \exp \left(-\frac{\Delta E}{k T}\right)+\frac{N_{b}}{\tau_{s k}}+\frac{N_{d}^{\prime}}{\tau_{b d}}+g^{\prime} \\
\frac{\mathrm{d} N_{d}}{\mathrm{~d} t}=-\frac{N_{d}}{\tau_{r}}-\frac{N_{d}}{\tau_{b d}}+\frac{N_{b}}{\tau_{b d}} \exp \left(-\frac{\Delta E}{k T}\right) \\
\frac{\mathrm{d} N_{d}^{\prime}}{\mathrm{d} t}=-\frac{N_{d}^{\prime}}{\tau_{r}}-\frac{N_{d}^{\prime}}{\tau_{b d}}+\frac{N_{b}^{\prime}}{\tau_{b d}} \exp \left(-\frac{\Delta E}{k T}\right),
\end{array}\right.
$$

The inter and intra valley scattering and recombination paths involved are summarized in figure1. In writing the rate equations we have assumed identical recombination times for the dark and bright excitons which is reasonable in TMDs due to the strong non radiative recombination [49, 50] which dominates over the radiative recombination of bright excitons. Under steady state conditions $(d N / d t=0)$ if we consider only the bright-dark exciton scattering in the rate equations we obtain $N_{b} / N_{d}=\exp (-\Delta E / k T)$ for $\mathrm{WX}_{2}$ and $N_{d} / N_{b}=\exp (-\Delta E / k T)$ for $\mathrm{MoX}_{2}$ which is simply the expected Boltzmann distribution. Thus, when the $\tau_{b d}$ is similar or shorter than $\tau_{r}$ (which is the case in TMDs i.e. $\tau_{b d} \ll 1 p s$ [51, 47, 48] and $\tau_{r}$ is in the range of picoseconds to hundreds of ps [24, 52, 53, 49, 50, 54, 55, 56, 23]) and the dark exciton is the ground state (in $\mathrm{WX}_{2}$ TMDs) it provides an important reservoir for valley polarization which tries to maintain the bright exciton population in the same valley (i.e. tries to maintain a Boltzmann distribution). This reservoir is robust since there is no exchange inter-valley scattering channel for dark excitons [45].

The observed degree of circular polarization in $\mathrm{PL}$ is related to the occupation of exciton bright states in $\mathbf{K}$ and $\mathbf{K}^{\prime}$ valleys,

$$
P=\frac{N_{b}-N_{b}^{\prime}}{N_{b}+N_{b}^{\prime}}
$$

To obtain the polarization we solve the rate equations assuming that excitons are generated only in $\mathbf{K}$ valley $\left(g^{\prime}=0\right)$, corresponding to $\sigma^{+}$excitation. The generation rate is assumed to be constant (cw excitation) and the polarization at steady state conditions $(d N / d t=0)$ is calculated. For simplicity all time constants for different processes are expressed in units of the exciton recombination time $\tau_{r}$ and the temperature is assumed to be $T=10 \mathrm{~K}$ (most of the PL literature data are taken close to this temperature). 


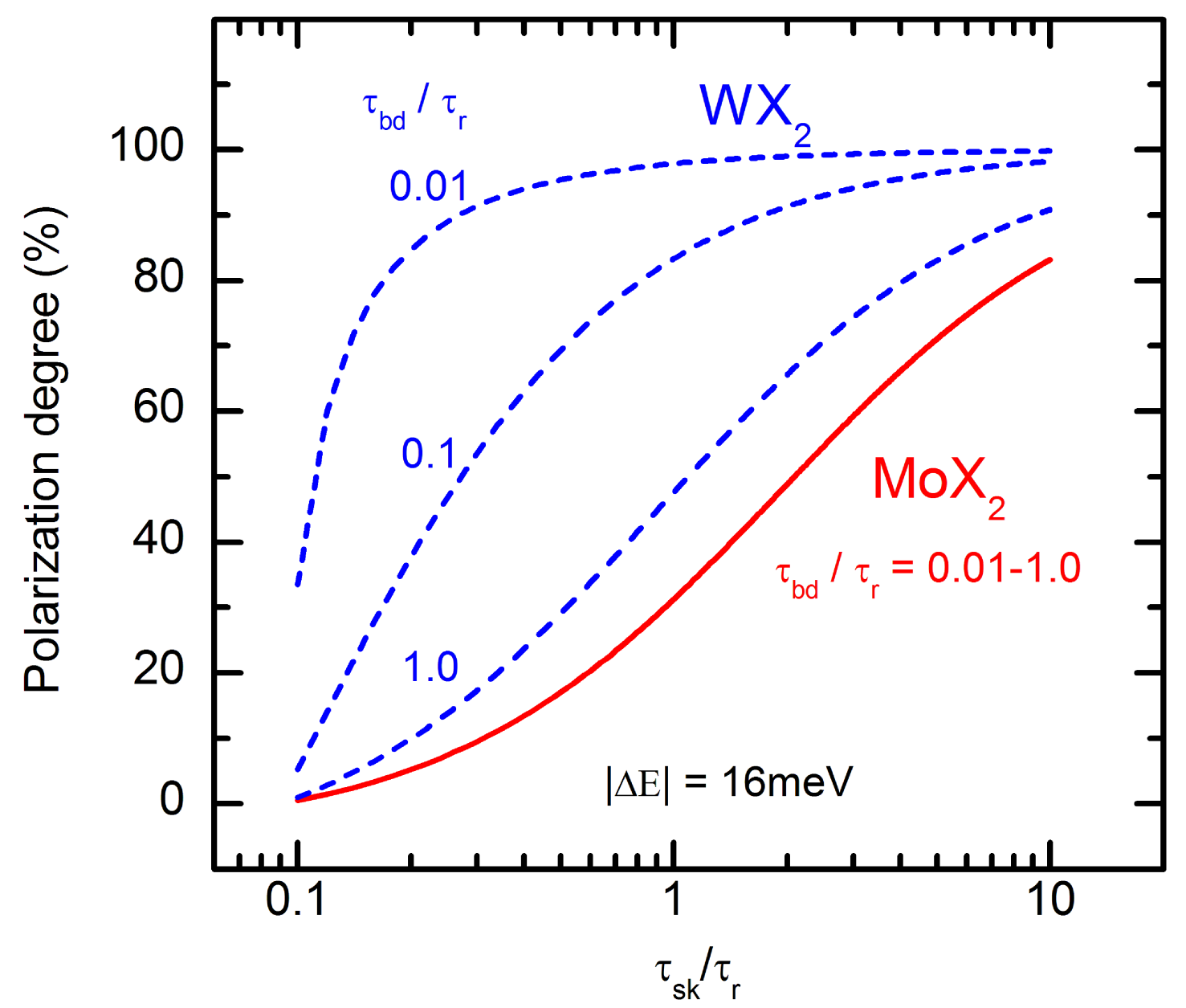

Figure 3. Degree of circular polarization in PL as a function of the inter-valley scattering time calculated using the rate equations for $\mathrm{WX}_{2}$ (broken lines) and $\mathrm{MoX}_{2}$ (solid lines) for three different values of the bright-dark exciton scattering time $\tau_{b d}$.

Figure 3 shows the calculated degree of polarization as a function of the intervalley scattering time $\tau_{s k}$ for $\mathrm{WX}_{2}$ and $\mathrm{MoX}_{2}$ TMDs, calculated for three different values of $\tau_{b d}$ assuming a typical bright-dark splitting of $\Delta E=16 \mathrm{meV}$. It is clearly seen that with decreasing $\tau_{s k}$ the degree of polarization decreases rapidly for both material systems as expected. However, the degree of polarization for TMDs with a dark exciton ground state $\left(\mathrm{WX}_{2}\right)$ is always higher than for TMDs with a bright exciton ground state $\left(\mathrm{MoX}_{2}\right)$. This has to be related to the different efficiency of the bright-dark exciton scattering channel, since the other terms in the rate equations are identical. Excitons are created only in the bright state and then scattered either to the other valley or to a dark exciton state in the same valley. Since the second process is highly efficient in $\mathrm{WX}_{2}$ (dark exciton ground state), it competes with inter-valley scattering. Thus, fewer excitons are scattered to the opposite valley, and the dark excitons constitute an important reservoir which tries to maintain $N_{b} / N_{d}$ close to the expected Boltzmann distribution. This increases the degree of polarization of the PL emission (and also 


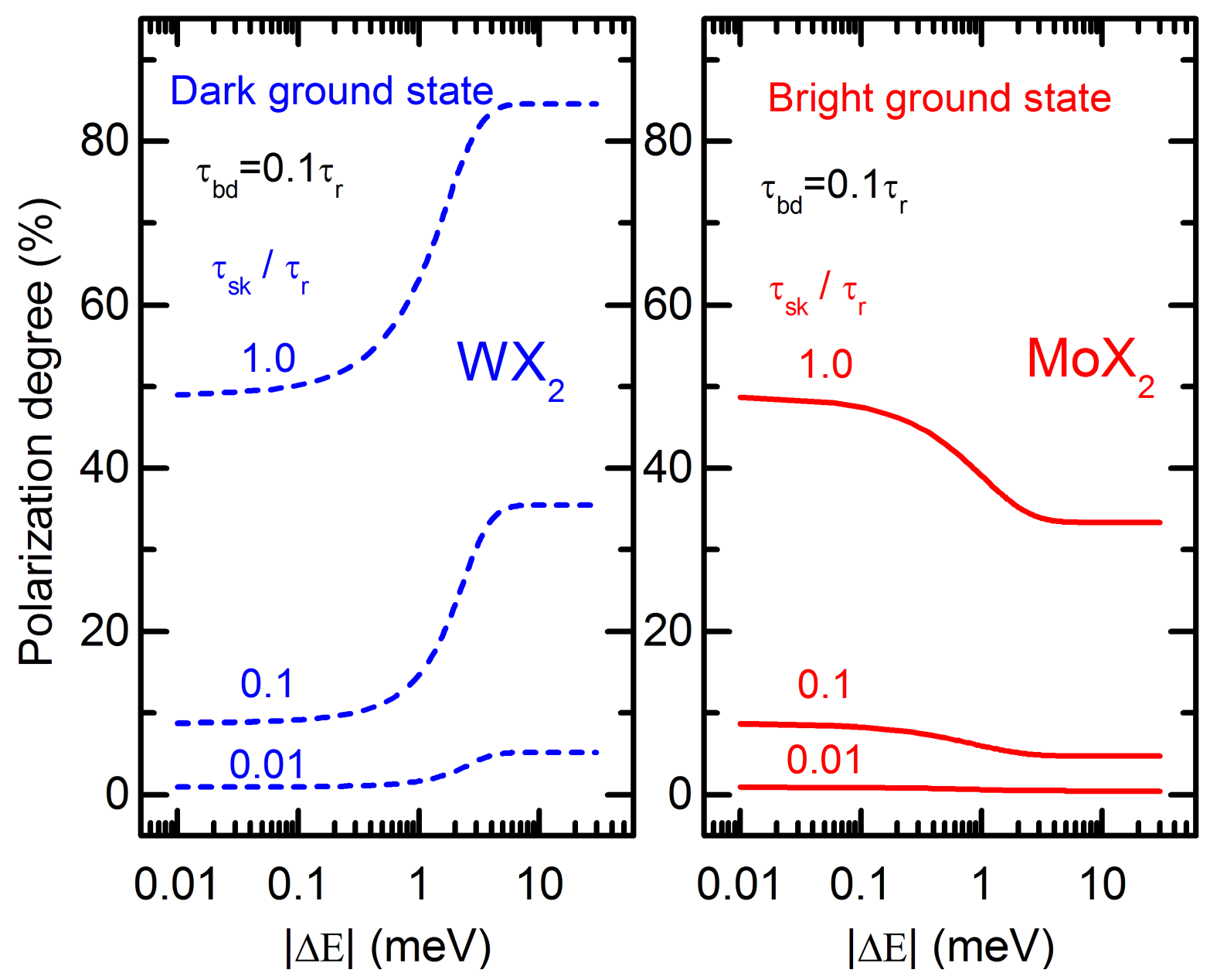

Figure 4. Dependence of PL circular polarization degree as function of absolute value of bright-dark exciton spin splitting calculated according to rate equations $3\left(\mathrm{WX}_{2}\right)$ and $4\left(\mathrm{MoX}_{2}\right)$ for different values of inter-valley scattering time.

the total valley polarization). For $\mathrm{MoX}_{2}$, the bright-dark exciton scattering is strongly damped and the inter-valley scattering dominates, which depletes the population of the optically excited valley, decreasing the degree of polarization of the PL emission. For MoX $\mathrm{X}_{2}$ TMDs, changes in the scattering time between bright and dark states do not affect the polarization degree (all lines overlap), the kinetics is dominated by inter-valley scattering at low temperature. On the other hand, the value of $\tau_{b d}$ significantly affects the polarization degree if the ground state is dark $\left(\mathrm{WX}_{2}\right)$. Fast bright-dark scattering (small value of $\tau_{b d}$ ) greatly enhances the degree of polarization especially if the intervalley bright exciton scattering is efficient $\left(\tau_{s k} / \tau_{r}<1\right)$. It is also notable that when the bright-dark scattering is efficient the polarization dependence exhibits a plateau for some range of $\tau_{s k}$ values. This is in agreement with experimental observation where for $\mathrm{WX}_{2}$ material system the degree of circular polarization is not so sensitive to the excitation energy (according to ref. [45] while the inter-valley scattering rate increases with exciton kinetic energy). 
Dark excitons and the elusive valley polarization in transition metal dichalcogenides 10

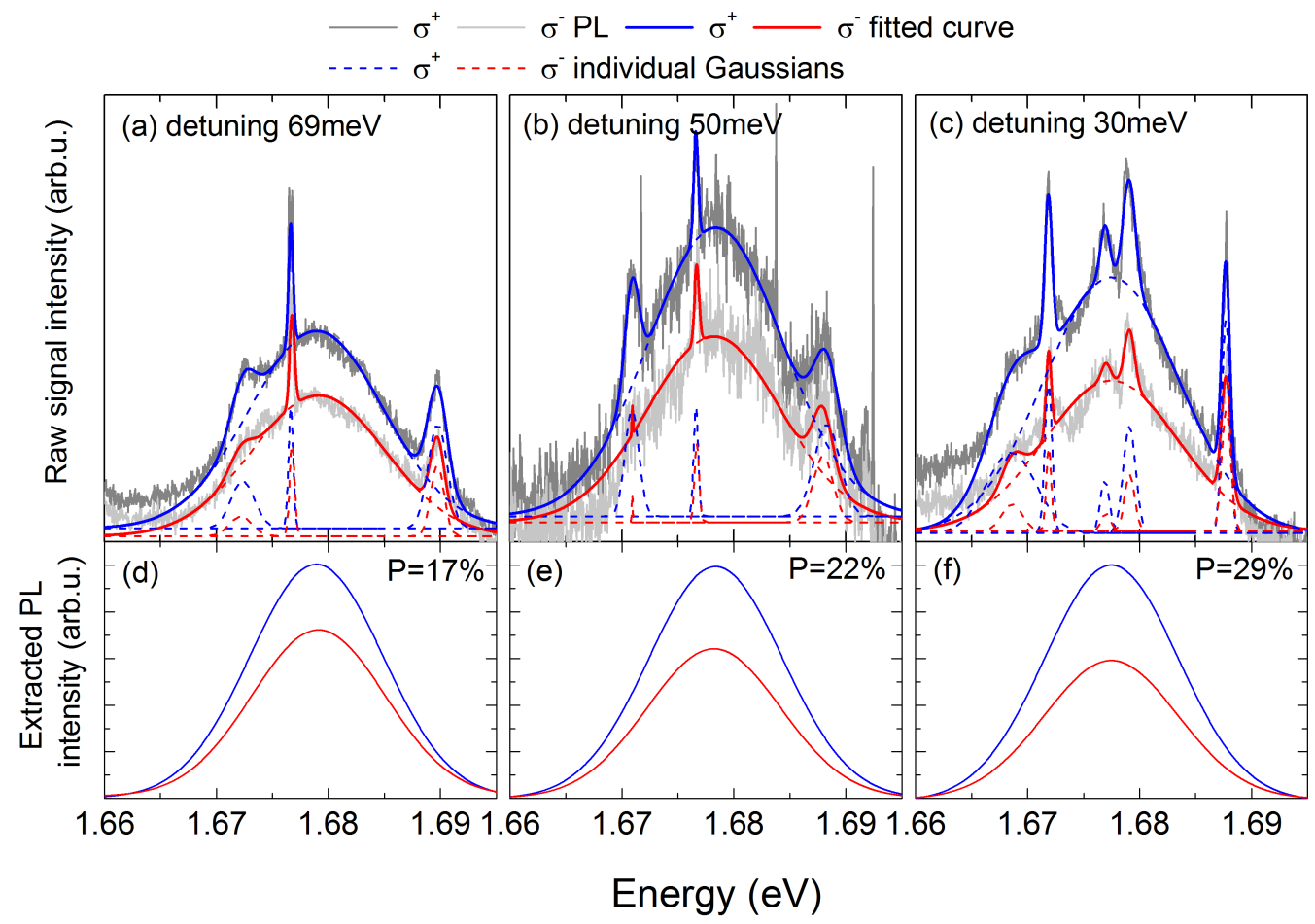

Figure 5. (a)-(c)Representative PL spectra obtained for $\sigma^{+}$(dark gray) and $\sigma^{-}$(light gray) polarization together with fitted curves (blue and red solid lines), the individual Gaussian components are plotted by dashed lines. (d)-(f) Extracted component related to PL of the A-exciton, blue (red) color indicates $\sigma^{+}\left(\sigma^{-}\right)$detection polarization. The degree of circular polarization $(P)$ calculated from the amplitudes of the $\sigma^{ \pm} \mathrm{PL}$ is indicated on each figure.

Figure 4 shows the dependence of the calculated PL polarization degree as a function of the absolute value of bright-dark exciton splitting for $\mathrm{WX}_{2}$ and $\mathrm{MoX}_{2}$. While the dark and bright states are degenerate $(|\Delta E| \ll k T)$ there is no difference in the PL polarization for either system. When the splitting between bright and dark states becomes non negligible, the PL circular polarization degree is significantly higher for the $\mathrm{WX}_{2}$ TMDs. With increasing bright-dark splitting the reservoir of dark states is increasingly populated and the degree of circular polarization in the PL emission increases. In contrast, in the $\mathrm{MoX}_{2}$ TMDs, increasing the bright-dark splitting reduces the population of the dark exciton reservoir and the degree of polarization of the PL emission actually decreases. This observation very well explains the observed dependence of PL circular polarization from the TMDs alloys such us $\mathrm{Mo}_{1-x} \mathrm{~W}_{x} \mathrm{Se}_{2}$ where the rapid increase of PL circular polarization is observed for increasing Tungsten content [57] and related to this realignment of bright and dark exciton states. 
Dark excitons and the elusive valley polarization in transition metal dichalcogenides 11

\section{Degree of circular polarization in Molybdenum diselenide}

A crucial test of the rate equation model and the pertinence of the assumption that the electron-hole exchange is the dominant inter valley scattering mechanism is provided by an investigation of the degree of polarization of the PL emission in $\mathrm{MoSe}_{2}$ which has a bright exciton ground state. For resonant excitation the two LA phonon model predicts $100 \%$ polarization while the rate equation model predicts significantly less, depending upon the bright-dark exciton scattering rate. For the measurements we have used a large area CVD grown $\mathrm{MoSe}_{2}$ monolayer encapsulated [58] with monolayer $\mathrm{MoS}_{2}$ to improve the optical quality [59].

For polarization resolved PL studies the sample was placed in a helium cryostat. All measurements were performed at $T \simeq 4.5 \mathrm{~K}$ and the sample was excited using a tunable CW Ti:sapphire laser. The wavelength of excitation was tuned from $710 \mathrm{~nm}$ to $730 \mathrm{~nm}$ (a much lower energy than the A-exciton transition in $\mathrm{MoS}_{2}$ encapsulating layers) and the excitation power was $\sim 800 \mu \mathrm{W}$. The excitation beam was focused on the sample via a $20 \times$ microscope objective ( 0.55 numerical aperture). The PL signal was collected through the same objective and dispersed by a triple grating monochromator equipped with 1800 grooves/mm grating and detected using a nitrogen cooled CCD detector. The excitation beam was circularly polarized using a linear polarizer combined with a Babinet-Soleil compensator. Using a zero-order quarter-wave plate and a subtractive mode linear polarizer, the PL signal was detected in $\sigma^{ \pm}$polarizations.

Representative PL spectra of the A-exciton emission detected in $\sigma^{+}$(dark grey line) and $\sigma^{-}$(light grey line) polarization are shown in figure5(a-c). Due to the small difference between the excitation and emission energy, the PL spectra exhibit, in addition to the A-exciton emission, a number of Raman peaks which progressively strengthen as resonant excitation is approached. To correctly analyze the degree of circular polarization of the A-exciton PL emission the Raman features have to be removed. To achieve this we first measure the PL far from resonances using a frequency doubled Nd:YAG laser emitting at $532 \mathrm{~nm}$. The PL spectra are then fitted using a Gaussian to extract the broadening and energy of the A-exciton recombination. These parameters are then fixed when fitting spectra measured close to resonant excitation using a number of Gaussian functions (depending on the number of Raman features observed). Fixing the energy and broadening of the A-exciton transition allows the amplitude to be reliably extracted. The results of the fitting procedure are shown by the blue $\left(\sigma^{+}\right.$polarization) and red $\left(\sigma^{-}\right.$polarization) curves in figure 5 (a-b) together with individual Gaussians (dashed lines). We have good agreement between fitted curves and experimental data. The extracted Gaussian peaks (normalized by the maximum value of $\sigma^{+}$PL signal) corresponding to the A exciton transition are presented in figure 5(d-f). The increase of the $\sigma^{+} \mathrm{PL}$ intensity relatively to $\sigma^{-}$is clearly visible with decreasing detuning i.e. the polarization degree increases. Based on the extracted PL intensities in both polarizations we determine the degree of PL circular polarization for all excitation wavelengths. The dependence of PL circular polarization versus detuning 
Dark excitons and the elusive valley polarization in transition metal dichalcogenides 12

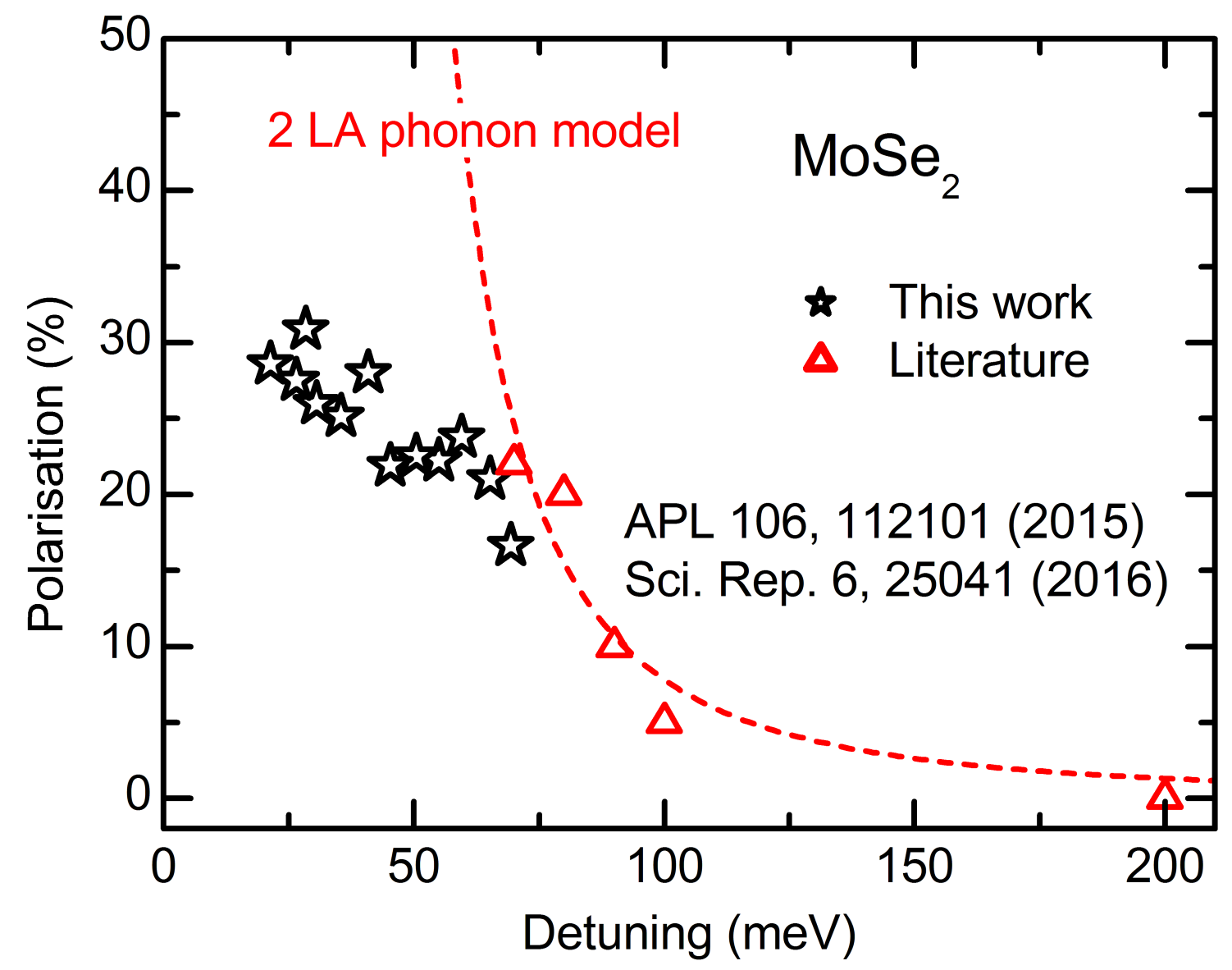

Figure 6. Measured degree of circular polarization of PL emission from $\mathrm{MoSe}_{2}$ as a function of detuning between excitation and exciton transition energy. Values at large detuning (triangles) are taken from the literature [30, 33. The dashed line is calculated dependence for two LA phonon model using $\hbar \omega_{2 L A}=38 \mathrm{meV}$ and $\alpha=0.1$.

energy is presented in figure 6. Black stars are our data and red triangles represent values reported for $\mathrm{MoSe}_{2}$ in the literature [33, 30]. While the literature data presents a rapid increase in the polarization for detuning energies below $\simeq 100 \mathrm{meV}$, our data reveals that this behavior rapidly saturates with an extrapolated (maximum) polarization for resonant excitation of $\simeq 35 \%$.

Finally, we compare the measured polarization with the predictions of the two LA phonon model [33],

$$
P=\left(1+\frac{\alpha \hbar \omega_{2 L A}}{\exp \left(\hbar \omega_{2 L A} /\left(E_{\text {det }}-\hbar \omega_{2 L A}\right)\right)-1}\right)^{-1},
$$

where $\hbar \omega_{2 L A}$ is twice the LA phonon energy, $E_{\text {det }}$ is the detuning and $\alpha \sim 1$ is a scaling constant. The dashed line in figure 6 is calculated using $\hbar \omega_{2 L A}=38 \mathrm{meV}$ and $\alpha=0.1$ which provides the best fit to the literature data. The two LA phonon model diverges asymptotically to $100 \%$ polarization as the detuning energy approaches $\hbar \omega_{2 L A}$ from above while the experimental data clearly saturates around the $35 \%$ for detunings below $\hbar \omega_{2 L A}$. This strongly suggests that the changes of the PL circular polarization degree 
with excitation wavelength are explained by the dependence of the exchange interaction on the exciton kinetic energy [45]. It is interesting to note that in case of $\mathrm{MoS}_{2}$ the PL circular polarization degree can reach almost $100 \%$ for small values of detuning indicating that inter-valley scattering $\left(1 / \tau_{s k}\right)$ is unable to destroy valley polarization if the excitons have low kinetic energy. In contrast, our experimental results suggest that $100 \%$ polarization cannot be achieved in $\mathrm{MoSe}_{2}$, a clear indication that inter-valley scattering remains effective even for excitons with low kinetic energy. These observations are consistent with the alignment of bright and dark exciton states in the Mo based TMDs. For $\mathrm{MoSe}_{2}$ all calculations predict that the bright exciton is the ground sate and the dark exciton is several meV above (see figure2). As we show such alignment results in attenuation of $\mathrm{PL}$ circular polarization. At the same time for $\mathrm{MoS}_{2}$ the exciton splitting is close to zero (see figure 2) so higher value of $P$ is expected (see figure 4) than in case of $\mathrm{MoSe}_{2}$.

\section{Conclusions}

In conclusion, we have developed a simple rate equation model for the excitons kinetics which is able to explain the differences in the observed degree of circular polarization of the PL emission in different TMDs monolayers. Our work suggests that the dark exciton states play an important, and previously unsuspected role in determining the degree of polarization of the PL emission. Inter valley scattering, which is mediated by the electron-hole exchange interaction, is effective only for bright exciton states. A dark exciton ground state provides a robust reservoir for valley polarization, which tries to maintain a Boltzmann distribution of the bright exciton states in the same valley via intra valley bright dark exciton scattering mechanism. This provides a simple explanation for the achievable polarization in TMDs. In $\mathrm{WX}_{2}$ TMDs in which the dark exciton is the ground state, $20-40 \%$ polarization can be achieved, even with non resonant excitation. The $\mathrm{MoX}_{2}$ TMDs with bright exciton ground states require almost resonant excitation to achieve the same polarization degree. The proposed model also explains the difference between different Molybdenum based materials as well as TMDs alloys. The dependence of the degree of polarization on the detuning energy of the excitation in $\mathrm{MoSe}_{2}$ suggests that the electron-hole exchange interaction dominates over two LA phonon emission mechanism for inter valley scattering in TMDs.

\section{Acknowledgments}

This work was partially supported by ANR JCJC project milliPICS, the Région MidiPyrénées under contract MESR 13053031, BLAPHENE project under IDEX program Emergence, HFML-RU/FOM, a member of the European Magnetic Field Laboratory (EMFL), STCU project 5809 and the Swiss SNF Sinergia Grant no. 147607. 
Dark excitons and the elusive valley polarization in transition metal dichalcogenides 14

[1] Wang Q H, Kalantar-Zadeh K, Kis A, Coleman J N and Strano M S 2012 Nature nanotechnology $7699-712$

[2] Xu X, Yao W, Xiao D and Heinz T F 2014 Nature Physics 10 343-350

[3] Zeng H and Cui X 2015 Chem. Soc. Rev. 44(9) 2629-2642

[4] Duan X, Wang C, Pan A, Yu R and Duan X 2015 Chem. Soc. Rev. 44(24) 8859-8876

[5] Liu G B, Xiao D, Yao Y, Xu X and Yao W 2015 Chem. Soc. Rev. 44(9) 2643-2663

[6] Mak K F, Lee C, Hone J, Shan J and Heinz T F 2010 Phys. Rev. Lett. 105(13) 136805

[7] Zeng H, Liu G B, Dai J, Yan Y, Zhu B, He R, Xie L, Xu S, Chen X, Yao W and Cui X 2013 Scientific Reports 31608

[8] Splendiani A, Sun L, Zhang Y, Li T, Kim J, Chim C Y, Galli G and Wang F 2010 Nano letters $101271-1275$

[9] Lopez-Sanchez O, Alarcon Llado E, Koman V, Fontcuberta i Morral A, Radenovic A and Kis A 2014 Acs Nano 8 3042-3048

[10] Jariwala D, Sangwan V K, Lauhon L J, Marks T J and Hersam M C 2014 ACS nano 8 1102-1120

[11] Ross J S, Klement P, Jones A M, Ghimire N J, Yan J, Mandrus D, Taniguchi T, Watanabe K, Kitamura K, Yao W et al. 2014 Nature nanotechnology 9 268-272

[12] Zhang Y, Oka T, Suzuki R, Ye J and Iwasa Y 2014 Science 344 725-728

[13] Mak K F and Shan J 2016 Nature Photonics 10 216-226

[14] Xiao D, Chang M C and Niu Q 2010 Reviews of modern physics 821959

[15] Xiao D, Liu G B, Feng W, Xu X and Yao W 2012 Physical Review Letters 108196802

[16] Cao T, Wang G, Han W, Ye H, Zhu C, Shi J, Niu Q, Tan P, Wang E, Liu B et al. 2012 Nature communications 3887

[17] Liu G B, Shan W Y, Yao Y, Yao W and Xiao D 2013 Physical Review B 88085433

[18] Yao W, Xiao D and Niu Q 2008 Physical Review B $\mathbf{7 7} 235406$

[19] He K, Kumar N, Zhao L, Wang Z, Mak K F, Zhao H and Shan J 2014 Physical review letters 113 026803

[20] Dhakal K P, Duong D L, Lee J, Nam H, Kim M, Kan M, Lee Y H and Kim J 2014 Nanoscale 6 13028-13035

[21] He K, Poole C, Mak K F and Shan J 2013 Nano letters 13 2931-2936

[22] Echeverry J, Urbaszek B, Amand T, Marie X and Gerber I 2016 Physical Review B 93121107

[23] Godde T, Schmidt D, Schmutzler J, Aßmann M, Debus J, Withers F, Alexeev E M, Del PozoZamudio O, Skrypka O V, Novoselov K S, Bayer M and Tartakovskii A I 2016 Phys. Rev. B 94(16) 165301

[24] Zhang X X, You Y, Zhao S Y F and Heinz T F 2015 Physical Review Letters 115257403

[25] Zeng H, Dai J, Yao W, Xiao D and Cui X 2012 Nature nanotechnology 7 490-493

[26] Mak K F, He K, Shan J and Heinz T F 2012 Nature nanotechnology 7 494-498

[27] Plechinger G, Nagler P, Kraus J, Paradiso N, Strunk C, Schüller C and Korn T 2015 physica status solidi (RRL)-Rapid Research Letters 9 457-461

[28] Scrace T, Tsai Y, Barman B, Schweidenback L, Petrou A, Kioseoglou G, Ozfidan I, Korkusinski $\mathrm{M}$ and Hawrylak P 2015 Nature nanotechnology 10 603-607

[29] Wang G, Bouet L, Lagarde D, Vidal M, Balocchi A, Amand T, Marie X and Urbaszek B 2014 Physical Review B $\mathbf{9 0} 075413$

[30] Wang G, Palleau E, Amand T, Tongay S, Marie X and Urbaszek B 2015 Applied Physics Letters 106112101

[31] Kioseoglou G, Hanbicki A, Currie M, Friedman A, Gunlycke D and Jonker B 2012 Applied Physics Letters 101221907

[32] Jones A M, Yu H, Ghimire N J, Wu S, Aivazian G, Ross J S, Zhao B, Yan J, Mandrus D G, Xiao D et al. 2013 Nature nanotechnology 8 634-638

[33] Kioseoglou G, Hanbicki A T, Currie M, Friedman A L and Jonker B T 2016 Scientific Reports 6 25041

[34] Zhu B, Zeng H, Dai J, Gong Z and Cui X 2014 Proceedings of the National Academy of Sciences 
Dark excitons and the elusive valley polarization in transition metal dichalcogenides 15

111 11606-11611

[35] Aivazian G, Gong Z, Jones A M, Chu R L, Yan J, Mandrus D G, Zhang C, Cobden D, Yao W and Xu X 2015 Nat Phys 11 148-152 ISSN 1745-2473

[36] Lagarde D, Bouet L, Marie X, Zhu C, Liu B, Amand T, Tan P and Urbaszek B 2014 Physical review letters 112047401

[37] Arora A, Schmidt R, Schneider R, Molas M R, Breslavetz I, Potemski M and Bratschitsch R 2016 Nano Lett. 16 3624-3629 ISSN 1530-6984

[38] Mai C, Barrette A, Yu Y, Semenov Y G, Kim K W, Cao L and Gundogdu K 2013 Nano letters $14202-206$

[39] Wang Q, Ge S, Li X, Qiu J, Ji Y, Feng J and Sun D 2013 ACS nano 7 11087-11093

[40] Zhu C, Zhang K, Glazov M, Urbaszek B, Amand T, Ji Z, Liu B and Marie X 2014 Physical Review B 90161302

[41] Dal Conte S, Bottegoni F, Pogna E, De Fazio D, Ambrogio S, Bargigia I, D'Andrea C, Lombardo A, Bruna M, Ciccacci F et al. 2015 Physical Review B 92235425

[42] Molina-Sanchez A and Wirtz L 2011 Physical Review B 84155413

[43] Horzum S, Sahin H, Cahangirov S, Cudazzo P, Rubio A, Serin T and Peeters F 2013 Physical Review B 87125415

[44] Terrones H, Del Corro E, Feng S, Poumirol J, Rhodes D, Smirnov D, Pradhan N, Lin Z, Nguyen M, Elias A et al. 2014 Scientific reports 44215

[45] Yu T and Wu M 2014 Physical Review B 89205303

[46] Glazov M, Amand T, Marie X, Lagarde D, Bouet L and Urbaszek B 2014 Physical Review B 89 201302

[47] Mai C, Semenov Y G, Barrette A, Yu Y, Jin Z, Cao L, Kim K W and Gundogdu K 2014 Physical Review B 90041414

[48] Sie E J, Frenzel A J, Lee Y H, Kong J and Gedik N 2015 Physical Review B 92125417

[49] Wang H, Zhang C and Rana F 2015 Nano Lett. 15 8204-8210 ISSN 1530-6984

[50] Wang H, Zhang C and Rana F 2015 Nano Lett. 15 339-345 ISSN 1530-6984

[51] Song Y and Dery H 2013 Physical review letters 111026601

[52] Schaibley J R, Karin T, Yu H, Ross J S, Rivera P, Jones A M, Scott M E, Yan J, Mandrus D, Yao W et al. 2015 Physical review letters 114137402

[53] Yan T, Qiao X, Liu X, Tan P and Zhang X 2014 Applied Physics Letters 105101901

[54] Korn T, Heydrich S, Hirmer M, Schmutzler J and Schüller C 2011 Applied Physics Letters 99 102109

[55] Robert C, Lagarde D, Cadiz F, Wang G, Lassagne B, Amand T, Balocchi A, Renucci P, Tongay S, Urbaszek B et al. 2016 Physical Review B 93205423

[56] Amani M, Lien D H, Kiriya D, Xiao J, Azcatl A, Noh J, Madhvapathy S R, Addou R, Santosh K, Dubey M et al. 2015 Science 350 1065-1068

[57] Wang G, Robert C, Suslu A, Chen B, Yang S, Alamdari S, Gerber I C, Amand T, Marie X, Tongay $\mathrm{S}$ et al. 2015 Nature communications 610110

[58] Wang K, Huang B, Tian M, Ceballos F, Lin M W, Mahjouri-Samani M, Boulesbaa A, Puretzky A A, Rouleau C M, Yoon M, Zhao H, Xiao K, Duscher G and Geohegan D B 2016 ACS Nano 10 6612-6622 ISSN 1936-0851

[59] Surrente A et al. 2016 The detailed sample preparation and optical caracterisation of the encapsulated samples will be the subject of another manuscript which is under preparation. 